\title{
BMJ Comparison of handheld rebound OPen tonometry with Goldmann applanation tonometry in children with glaucoma: a cohort study
}

\author{
Annegret Hella Dahlmann-Noor, ${ }^{1}$ Renata Puertas, ${ }^{2}$ Shenille Tabasa-Lim, ${ }^{1}$ \\ Ahmed El-Karmouty, ${ }^{2}$ Mustafa Kadhim, ${ }^{1}$ Nicholas Kloster Wride, ${ }^{2}$ Amanda Lewis, ${ }^{2}$ \\ Dawn Grosvenor, ${ }^{2}$ Poornima Rai, ${ }^{2}$ Maria Papadopoulos, ${ }^{2}$ John Brookes, ${ }^{1}$ \\ Catey Bunce, ${ }^{3}$ Peng Tee Khaw ${ }^{2}$
}

To cite: Dahlmann-Noor AH, Puertas R, Tabasa-Lim S, et al. Comparison of handheld rebound tonometry with Goldmann applanation tonometry in children with glaucoma: a cohort study. BMJ Open 2013;3:e001788. doi:10.1136/bmjopen-2012001788

- Prepublication history for this paper are available online. To view these files please visit the journal online (http://dx.doi.org/10.1136/ bmjopen-2012-001788).

Received 25 July 2012 Revised 11 November 2012 Accepted 26 November 2012

This final article is available for use under the terms of the Creative Commons Attribution Non-Commercial 2.0 Licence; see http://bmjopen.bmj.com

For numbered affiliations see end of article.

Correspondence to Dr Annegret Dahlmann-Noor; annegret.dahlmann-noor@ moorfields.nhs.uk

\section{ABSTRACT}

Objective: To test agreement of two methods to measure intraocular pressure (IOP): rebound tonometry (RBT) and gold standard Goldmann applanation tonometry (GAT) in children with glaucoma.

Design: Observational prospective cohort study.

Setting: Tertiary paediatric glaucoma clinic at a single centre.

Participants: 102 individuals attending a paediatric glaucoma clinic, mean (SD) age 11.85 (3.17), of whom 53 were male.

\section{Primary and secondary outcome measures:}

Intraocular pressure, central corneal thickness, child preference for measurement method.

Results: Limits of agreement for intraobserver and interobserver were, respectively, $(-2.71,2.98) \mathrm{mm} \mathrm{Hg}$ and $(-5.75,5.97) \mathrm{mm} \mathrm{Hg}$. RBT frequently gave higher readings than GAT and the magnitude of disagreement depend on the level of IOP being assessed. Differences of $10 \mathrm{~mm} \mathrm{Hg}$ were not uncommon. RBT was the preferred method for $70 \%$ of children.

Conclusions: There is poor agreement between RBT and GAT in children with glaucoma. RBT frequently and significantly overestimates IOP. However, 'normal' RBT readings are likely to be accurate and may spare children an examination under anaesthesia (EUA). High RBT readings should prompt the practitioner to use another standard method of IOP measurement if possible, or consider the RBT measurement in the context of clinical findings before referring the child to a specialist clinic or considering EUA.

\section{INTRODUCTION}

Glaucoma is a potentially blinding condition. Raised intraocular pressure (IOP) is an important risk factor for the development and progression of optic nerve damage in glaucoma, and is the target of both medical and surgical treatment. There are no published figures on how many children have an IOP test

\section{ARTICLE SUMMARY}

Article focus

- Measuring intraocular pressure (IOP) is critical in diagnosis and to monitor glaucoma, and is often difficult in children.

- Rebound tonometry (RBT) may be more child -friendly than other tonometry methods.

- Agreement between RBT and reference standard Goldmann applanation tonometry (GAT) in children and young people attending glaucoma clinics has not been sufficiently evaluated.

Key messages

- Agreement between RBT and GAT is poor in children and young people with glaucoma; RBT frequently and significantly overestimates IOP.

- 'Normal' RBT readings are likely to be accurate and may spare children for an examination under anaesthesia.

- High RBT readings should prompt the practitioner to use another standard method of IOP measurement, if possible.

Strengths and limitations of this study

- This study focuses on a cohort of children and young people attending a paediatric glaucoma clinic, a population in whom accurate tonometry is required for correct diagnosis and management.

- Statistical analysis is based on the limits of agreement method rather than correlation. 
version of GAT, is a suitable reference standard for IOP measurements for patients in the supine position, and hence for children during an examination under anaesthesia. Another commonly used handheld tonometer is the Reichert Tono-Pen, but for comparison of diagnostic test accuracy, we opted for the reference standard devices.

GAT requires the instillation of topical anaesthetics and a high degree of cooperation from the child. Frequently, children are unable to suppress the natural reflex of moving backward or closing their eyes. This leads to repeated attempts being made at obtaining a reading, prolonged examination times and distress to children and families.

In 2005 a new device became available to measure IOP, the iCare rebound tonometer (RBT). Its main advantage over other instruments is that no topical anaesthesia is required. In addition, it is a handheld device, which children may perceive as less intimidating and allows examiners to move 'with the child' when required.

The RBT acquires six readings in quick succession by propelling a fine sensor tip less than $2 \mathrm{~mm}$ in diameter against the central cornea from the instrument base held at a distance of 4-8 $\mathrm{mm}$ from the surface of the eye. ${ }^{1}{ }^{2}$ ) After contact, the probe bounces back; the higher the IOP the faster the rebound. Measurements are taken within $0.1 \mathrm{~s}$. The force impacting on the cornea is minimal and usually does not even trigger the highly sensitive blink reflex. The instrument display then shows the average of the six readings and whether the $\mathrm{SD}$ is low, medium or high.

Since its introduction, RBT has become very popular and is now being used in many children's eye services and by many community-based optometrists. Two surveys of tonometry practice in children (table 1 and ref. ${ }^{3}$ ) indicate that for most paediatric ophthalmologists in the UK, RBT has become the preferred method to measure IOP in children. While the device is considered easy to use, many users report that RBT measurements tend to be higher than GAT measurements (table 1).

Data comparing RBT performance with GAT in children with glaucoma are scarce and limited to one study only. ${ }^{4}$ Studies which have compared RBT with noncontact tonometry and tonopen in healthy children indicate good intraobserver and interobserver reliability and good agreement. ${ }^{5-7}$ In children with glaucoma good agreement has been reported with both $\mathrm{PAT}^{8}$ and GAT, although RBT measurements are consistently higher than PAT or GAT readings.

In adults with healthy eyes, glaucoma or ocular hypertension, papers have reported good agreement between RBT and GAT, albeit with systematically higher measurements on RBT. ${ }^{9-15}$

Accuracy of RBT may be affected by levels of IOP, ${ }^{8} 16$ central corneal thickness (CCT) ${ }^{71314}$ and other corneal properties such as hysteresis and resistance. ${ }^{17}$ The latter parameters are not routinely assessed in clinics and impossible to assess in young children.

We carried out the present study to evaluate the accuracy and reliability of RBT in children with glaucoma as compared to GAT. Secondary objectives were to investigate the influence of CCT on IOP measurements by RBT, and to determine children's preference for RBT or GAT.

\section{METHODS}

Patients

We recruited consecutive participants attending our tertiary paediatric glaucoma service at Moorfields Eye
Table 1 Survey of current paediatric tonometry practice in the UK

In which setting do you work and measure IOP in children?

Do you run a specialist paediatric glaucoma service?

In how many children do you measure the IOP per month?

What is your preferred method to measure IOP in children?

If you are using an RBT, how easy do you find it to use?

In your experience, are RBT readings accurate as compared to GAT readings?

Teaching hospital
District general hosp
Community clinic
Yes
No
Less than 5
Between 5 and 20
More than 20
RBT

Goldmann/Perkins applanation

Tonopen

Air puff

Very easy

Moderately easy

Not at all easy

Not applicable

As accurate as Goldmann

RBT tend to be higher than GAT readings

RBT tend to be lower than GAT readings

Using an electronic mailing list, we contacted 144 paediatric ophthalmologists in the UK. The survey ran for 10 days, from 2 February 2012 to 16 February 2012, and collected 36 replies.

GAT, Goldmann applanation tonometer; IOP, intraocular pressure; RBT, rebound tonometer. Bold represents significant replies about RBT preference, ease of use, and limitations. 
Table 2 Demographic factors of the children under investigation

\begin{tabular}{|c|c|c|}
\hline Sex & Male:female $(n)$ & $53: 49$ \\
\hline Age (years) & Mean (SD) & $11.85(3.17)$ \\
\hline Laterality & Right:left & $85: 17$ \\
\hline \multirow[t]{6}{*}{ Ethnicity } & Caucasian & 57 \\
\hline & Mixed & 19 \\
\hline & Asian or Asian British & 1 \\
\hline & Caribbean/African/any other Black background & 8 \\
\hline & Other ethnic groups (including Chinese) & 11 \\
\hline & Not stated or not recorded & 6 \\
\hline Pachymetry & Median (IQR)* & $581(537,622)$ \\
\hline
\end{tabular}

Hospital. The study was approved by the local research ethics committee. All parents/guardians gave written informed consent and children were invited to give assent.

The sample size was determined by recommendations for Bland-Altman analysis. ${ }^{18}$

\section{Protocol}

Three observers took the measurements required for this study. Observer 1 measured the IOP in one eye by RBT. In children with glaucoma in both eyes, readings were taken from the right eye. In children with glaucoma-only in the left eye, readings were taken from the left eye. Observer 1 recorded two consecutive average RBT readings from six measurements and the SD indicated by the device.

One repeat average RBT measurement was obtained by a second observer and recorded separately.

Following instillation of a drop of proxymethacaine, observer 2 recorded CCT on ultrasound pachymetry.

The third observer recorded IOP on GAT and asked the child which instrument they preferred.

All data were recorded on a case report form which also recorded the child's diagnosis and ethnic background. At the end of the study, data were entered into a computerised database.

\section{Data analysis}

Summary statistics were produced for demographic factors and for IOP readings made by each observer and method (tables 2 and 3). Repeat readings made by observer 1 using RBT were used to estimate intraobserver agreement using the Bland-Altmann limits of agreement method. ${ }^{18}$ The same method was used to determine interobserver agreement, analysing RBT reading 1 from observer 1 and the RBT reading obtained by observer 2. Agreement between RBT and GAT was analysed based on reading 1 from observer 1 and GAT recorded by observer 3. A histogram for CCT was plotted in two age bands (under and over 10 years of age). Child preference for either device was summarised by descriptive statistical methods.

\section{RESULTS \\ Demographics}

We carried out the study between 1 January 2009 and 31 March 2010. We enrolled $n=102$ children with a confirmed diagnosis of glaucoma. Age ranged from 4.9 to 19 years (mean 11.85 , SD 3.17 years). A total of 53 boys and 49 girls took part (table 2). In 85 children the right eye was the study eye, and in $n=17$ children it was the left eye.

\section{Analysis of first RBT reading by observer 1}

In total 102 datasets were analysed. Data were missing for three participants. The RBT automatically indicates a higher-than-normal SD of the acquired measurements by displaying a flashing ' $\mathrm{P}$ ' or a line in the 'Up' position following the measurement. These are indicators of poor reliability, and the manufacturer recommends repeating the measurement. We excluded 16 participants in whom measurements displayed these poor reliability parameters. Nineteen sets of data were therefore excluded from analysis.

\section{Analysis of second RBT reading by observer 1}

There were nine missing values, and 11 children with poor reliability scores. Cross-tabulation of the first

Table 3 Agreement (interobserver, intraobserver and with GAT) for RBT

\begin{tabular}{|c|c|c|c|}
\hline & Mean difference & SD difference & LOA \\
\hline Intraobserver $(n=74)$ & 0.13 & 1.46 & $(-2.71,2.98)$ \\
\hline Interobserver $(n=45)$ & 0.11 & 2.99 & $(-5.75,5.97)$ \\
\hline ICare GAT $(n=74)$ & -3.34 & 5.31 & $\begin{array}{l}<21 \mathrm{~mm} \mathrm{Hg}(-8.60,3.90) \\
\geq 21 \mathrm{~mm} \mathrm{Hg}(-21.08,10.04)\end{array}$ \\
\hline
\end{tabular}


and second reading by observer 1 showed that readings were missing on both occasions for three children, and missing only on the second occasion for six children. Six children had poor reliability indicators on both the measurements.

\section{Analysis of RBT reading by observer 2}

Data were missing for 38 children. This was owing to the trial being run within a busy tertiary centre clinic, with observers not always able to complete this part of the examination.

Excluding RBT data with poor reliability indicators, we were left with 83 measurements by observer 1 (reading 1), 82 measurements by observer 1 (reading 2) and 50 measurements by observer 2 .

\section{Analysis of GAT data}

GAT readings were missing in 12 datasets. Most of these children had complex ocular pathology (corneal graft $\mathrm{n}=1$, microphthalmos/sunken socket $\mathrm{n}=1$, nystagmus $\mathrm{n}=1$, microcornea $\mathrm{n}=1$, congenital glaucoma and multiple surgery $n=1$, Sturge-Weber syndrome $n=1$ ). One child was very anxious and difficult to examine. Two children were glaucoma suspects, and in three cases no comment was available explaining missing GAT.

\section{Analysis of IOP readings by RBT and GAT}

The mean RBT reading 1 (SD) by observer 1 was $21.1 \mathrm{~mm} \mathrm{Hg}$ (8.19), RBT reading 2 by observer 1 was $21.9 \mathrm{~mm} \mathrm{Hg}$ (8.94), observer $2 \mathrm{RBT}$ was $21.14 \mathrm{~mm} \mathrm{Hg}$ (8.41) and GAT was $18 \mathrm{~mm} \mathrm{Hg}$ (6.45).

\section{Reproducibility of RBT readings: intraobserver and interobserver variability}

Data were available for 74 children who had two readings by observer 1 . The mean difference between the readings was 0.135 (SD 1.45). A t test revealed no evidence of systematic bias between readings 1 and 2 $((73 \mathrm{df})=0.7987, \mathrm{p}=0.427)$.

The distribution of the differences appeared symmetric. The Bland-Altman plot showed no systematic relationship between the average measure and the difference, and limits of agreement were computed as (-2.71, 2.98) $\mathrm{mm} \mathrm{Hg}$ (figure 1A).

First RBT reading by observer 1 and RBT reading by observer 2 were available for 45 cases. The mean difference between readings was $0.11 \mathrm{~mm} \mathrm{Hg}$ (2.99). A t test revealed no evidence of systematic bias between observers $(\mathrm{t}(44)=0.25, \mathrm{p}=0.8)$.

Again, the data appeared symmetric. The Bland-Altman plot showed no systematic relationship, limits of agreement were $(-5.75,5.97) \mathrm{mm} \mathrm{Hg}$ (figure $1 \mathrm{~B})$.

\section{Agreement between GAT and RBT}

We used data from first RBT reading by observer 1. These and GAT data were available for 74 children. The mean difference was $-3.3 \mathrm{~mm} \mathrm{Hg}$ (5.3). A t test showed evidence of systematic bias $(\mathrm{t}(73)=-5.41$, $\mathrm{p}<0.001)$. The Bland-Altman plot also showed evidence of a relationship between the magnitude of the difference between methods and the magnitude of the measurement, with smaller differences being seen with lower measurements. This would imply that limits of agreement cannot be simply produced. Stratifying data at $21 \mathrm{~mm} \mathrm{Hg}$ removed evidence of bias and limits of agreement were then computed as $(-8.6,3.9) \mathrm{mm} \mathrm{Hg}$ for readings less than $21 \mathrm{~mm} \mathrm{Hg}$ and $(-21.08,10.04)$ $\mathrm{mm} \mathrm{Hg}$ for readings greater than $21 \mathrm{~mm} \mathrm{Hg}$.

Based on these analyses, agreement between the two methods appears poor and not clinically acceptable.

As demonstrated by the graph (figure 1C), RBT frequently gave higher readings than GAT. Differences of $10 \mathrm{~mm} \mathrm{Hg}$ were not uncommon.

\section{Pachymetry data}

Pachymetry measurements were obtained in $n=83$ children, of whom 67 had IOP measurements taken by both RBT and GAT (figure 1D). Defining the 'normal range' as $460-650 \mu \mathrm{m}, 2$ readings were below the normal range $(452,456 \mu \mathrm{m})$ and 13 readings were above the normal range; of these, seven participants had both RBT and GAT measurements. Of the 13 children with CCT greater than $650 \mu \mathrm{m}$, one had corneal clouding/oedema and enlarged cornea, and in two children 'multiple previous glaucoma interventions' were recorded. None of these children had nystagmus, aphakic glaucoma, aniridia or anterior segment dysgenesis syndromes, or other confounding corneal abnormalities.

\section{Pachymetry and RBT readings}

Disagreement between GAT and RBT readings was greater with higher pachymetry readings, $\mathrm{p}=0.003$.

\section{Patient preference}

Eleven children preferred GAT, 70 preferred RBT and 21 gave no preference.

\section{DISCUSSION}

The main finding of the present study is that the agreement of RBT and GAT appears poor in children with glaucoma. Since previous studies have largely concluded that there is a good agreement between RBT and applanation tonometry, this observation was a surprise.

We believe that this discrepancy may be in part because of the fact that many studies have considered correlation rather than agreement. It is quite possible for methods to correlate well, but to disagree by amounts that are of real clinical relevance. Our data provide strong evidence of a linear association between measures made by GAT and RBT, but linear association is not the same as agreement, which is why Bland and Altman advocated the Limits of Agreement method. ${ }^{18}$

The present study is the second to compare RBT and GAT in children with glaucoma. The only other study in RBT in this population also included children with 

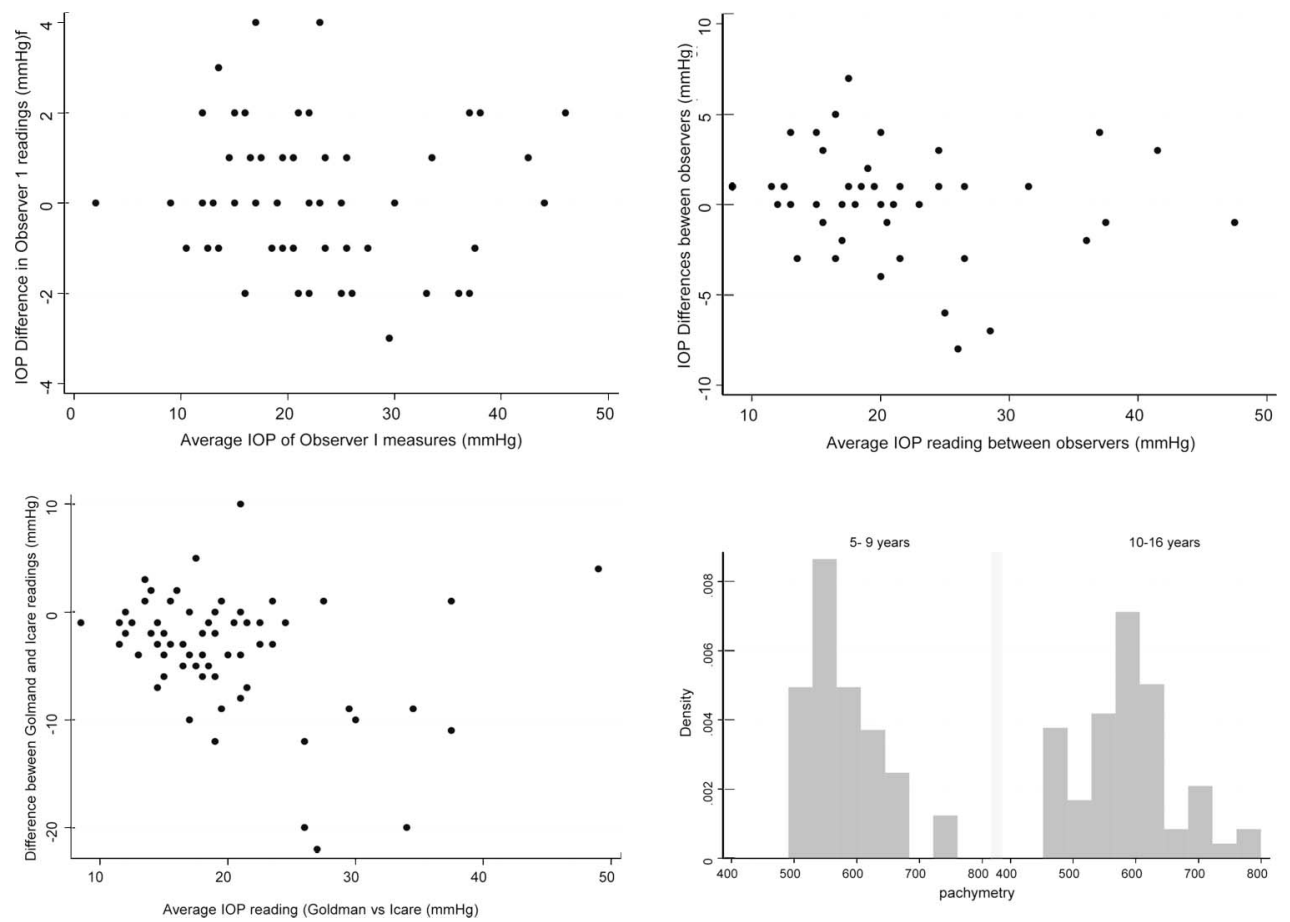

Figure 1 Tonometry and pachymetry results. (A) Intraobserver agreement of rebound tonometer (RBT) measurements; (B) interobserver agreement of RBT measurements; (C) agreement between RBT and Goldmann applanation tonometer measurements and (D) distribution of corneal thickness as determined by pachymetry. In healthy children of age 5-9 years, mean (SD) central corneal thickness has been reported as $566(48) \mu \mathrm{m}$ and in children over the age of 10 years, $554(35) \mu \mathrm{m} .{ }^{27}$

suspected glaucoma, excluded readings from children who were nervous or squeezing during the RBT measurement, and repeated RBT readings until a measurement with minimal variability was obtained, or three readings had been taken. ${ }^{4}$ While this guaranteed the vast majority of readings had either minimal or low variability, this approach means that the operator manually reduced device errors. The results of this study are comparable to those presented in our work: the authors report that RBT were higher than GAT readings in $75 \%$, and within $\pm 3 \mathrm{~mm} \mathrm{Hg}$ of GAT in $63 \%$. ${ }^{4}$ Our view is that disagreements of greater than $3 \mathrm{~mm} \mathrm{Hg}$ are of concern if they can impact on the management of the child.

The only other study exploring the use of RBT in children with glaucoma compared with PAT. ${ }^{8}$ The authors performed RBT and PAT in random order, which means that RBT measurements were performed after the administration of topical anaesthesia. According to the manufacturer's manual topical anaesthesia may reduce the RBT reading. 1920

Again, the authors repeated RBT measurements when the variability indicator was high or IOP $>19 \mathrm{~mm} \mathrm{Hg}$ with medium standard variation, excluding readings with erroneous device performance. The authors found greater disagreement with higher IOP measurements, yet did not provide limits of agreement stratified by IOP, or present regression-based limits of agreement. Interestingly, the graphs show that RBT measurements were consistently higher, up to $17 \mathrm{~mm} \mathrm{Hg}$, which is consistent with our findings. Both $\mathrm{PAT}$ and $\mathrm{RBT}$ readings were higher in cases of increased corneal thickness, another finding that agrees with our report.

Other paediatric studies of RBT are limited to healthy children, and none used GAT as comparator. ${ }^{5621}$

Difference in study population is likely to be a contributory factor explaining why agreement between RBT and GAT was significantly poorer in our study than in others. Comparisons with GAT have been conducted in adults with glaucoma, but excluding other significant pathologies such as astigmatism, microphthalmos, buphthalmos, dry eyes and nystagmus. ${ }^{11} 131522$ Results in studies with mixed populations (adults with glaucoma/ocular hypertension and healthy adults) also reported good GAT/RBT agreement. ${ }^{12} 1623$

However, all studies except two used topical anaesthesia before RBT. Interestingly, the two studies that did not use topical anaesthesia found significant differences between GAT and RBT, with RBT overestimating IOP by 1-15 mm Hg. ${ }^{15} 16$

Three studies performed on healthy adults without topical anaesthesia reported good agreement between RBT and GAT, ${ }^{9} 1024$ while three others reported significant differences in some cases. ${ }^{14} 1725$ The main limitation of these studies might be the inclusion of 'normal' eyes with normal IOP only. Findings from this population cannot be generalised to participants with raised IOP.

Sequence of tests may also explain differences in findings. Applanation tonometry, especially repeated measurements, can progressively lower IOP by a mechanism know as 'aqueous massage'. ${ }^{12} 1626$ Our study design 
avoided this effect by obtaining RBT before GAT measurements. Studies that obtained GAT before RBT or conducted tests in random order ${ }^{8} 11 \quad 132223$ may have induced IOP lowering, thereby erroneously obtaining lower RBT readings.

Similar to other groups 8131622 we found increased disagreement (ie, larger discrepancies in IOP measures between RBT and GAT with higher CCT values. However, we found that even in children with pachymetry readings of $581 \mu \mathrm{m}$, disagreements could be greater than $10 \mathrm{~mm} \mathrm{Hg}$.

Our study confirms reports ${ }^{5} 621$ that the handheld RBT has high acceptability in children. Since we started this work in 2008, it has become widely available and popular in many community optometric practices and hospital eye units in the UK (table 1). ${ }^{3}$ Seventy per cent of the children enrolled in this study had a clear preference for the new device which does not require instillation of anaesthetic eye drops and no prolonged sitting in an uncomfortable position.

On the basis of previously published reports and our own work, we would recommend the use of RBT in children, but advise practitioners to be cautious in the interpretation of findings, particularly in children with thicker corneas. It is reassuring that measurement discrepancies tend to reflect a systematic over-reading by the RBT: 'normal' values obtained by this method are likely correct, and the IOP is unlikely to be high. This in turn can reassure examiners and spare children the need for other IOP measurement methods and even examinations under anaesthesia. High RBT readings, on the other side, should not automatically trigger a trip to the operating theatre, but should prompt the examiner to seek confirmation of IOP level by another established technique.

\section{Author affiliations \\ ${ }^{1}$ Department of Paediatric Ophthalmology, NIHR Biomedical Research Centre at Moorfields Eye Hospital NHS Foundation Trust and UCL Institute of Ophthalmology, London, UK \\ ${ }^{2}$ Glaucoma, NIHR Biomedical Research Centre at Moorfields Eye Hospital NHS Foundation Trust and UCL Institute of Ophthalmology, London, UK ${ }^{3}$ Research and Development, Moorfields Eye Hospital NHS Foundation Trust, London, UK}

Acknowledgements The authors are grateful for the support from Fight for Sight and the Helen Hamlyn Trust. The research took place at the National Institute for Health Research (NIHR) Biomedical Research Centre at Moorfields Eye Hospital NHS Foundation Trust and UCL Institute of Ophthalmology. The views expressed are those of the author(s) and not necessarily those of the NHS, the NIHR or the Department of Health.

Contributors $A H D N$ and $C B$ designed the trial and prepared the manuscript. AHDN, RP, STL, AEK, PR, MK, NKW, AL, DG, MP, JB and PTK facilitated recruitment, obtained informed consent, acquired data and completed case report forms. AHDN entered data onto the electronic database. CB analysed the data. All the authors reviewed the manuscript.

Funding This research received no specific grant from any funding agency in the public, commercial or not-for-profit sectors.

Competing interests None.

Patient consent Obtained.
Ethics approval Royal Free Hospital and Medical School.

Provenance and peer review Not commissioned; externally peer reviewed.

Data sharing statement There are no additional data available.

\section{REFERENCES}

1. Kontiola Al, Goldblum D, Mittag T, et al. The induction/impact tonometer: a new instrument to measure intraocular pressure in the rat. Exp Eye Res 2001;73:781-5.

2. Kontiola A, Puska P. Measuring intraocular pressure with the Pulsair 3000 and Rebound tonometers in elderly patients without an anesthetic. Graefes Arch Clin Exp Ophthalmol 2004;242:3-7.

3. Chan WH, Lloyd IC, Ashworth JL, et al. Measurement of intraocular pressure in children in the UK. Eye 2012;25:119-20.

4. Flemmons MS, Hsiao YC, Dzau J, et al. ICare rebound tonometry in children with known and suspected glaucoma. J AAPOS 2011;15:153-7.

5. Kageyama M, Hirooka K, Baba $\mathrm{T}$, et al. Comparison of ICare rebound tonometer with noncontact tonometer in healthy children. J Glaucoma 2011;20:63-6.

6. Sahin A, Basmak H, Niyaz L, et al. Reproducibility and tolerability of the ICare rebound tonometer in school children. J Glaucoma 2007;16:185-8.

7. Sahin A, Basmak $H$, Yildirim $N$. The influence of central corneal thickness and corneal curvature on intraocular pressure measured by tono-pen and rebound tonometer in children. $J$ Glaucoma 2008;17:57-61.

8. Martinez-de-la-Casa JM, Garcia-Feijoo J, Saenz-Frances F, et al. Comparison of rebound tonometer and Goldmann handheld applanation tonometer in congenital glaucoma. J Glaucoma 2009;18:49-52.

9. Fernandes P, Diaz-Rey JA, Queiros A, et al. Comparison of the ICare rebound tonometer with the Goldmann tonometer in a normal population. Ophthalmic Physiol Opt 2005;25:436-40.

10. Davies LN, Bartlett $\mathrm{H}$, Mallen EA, et al. Clinical evaluation of rebound tonometer. Acta Ophthalmol Scand 2006;84:206-9.

11. Brusini $P$, Salvetat ML, Zeppieri M, et al. Comparison of ICare tonometer with Goldmann applanation tonometer in glaucoma patients. J Glaucoma 2006;15:213-17.

12. Nakamura M, Darhad U, Tatsumi $Y$, et al. Agreement of rebound tonometer in measuring intraocular pressure with three types of applanation tonometers. A J Ophthalmol 2006;142:332-4.

13. Sahin A, Niyaz L, Yildirim N. Comparison of the rebound tonometer with the Goldmann applanation tonometer in glaucoma patients. Clin Experiment Ophthalmol 2007;35:335-9.

14. Pakrou N, Gray T, Mills R, et al. Clinical comparison of the ICare tonometer and Goldmann applanation tonometry. J Glaucoma 2008;17:43-7.

15. Rehnman JB, Martin L. Comparison of rebound and applanation tonometry in the management of patients treated for glaucoma or ocular hypertension. Ophthalmic Physiol Opt 2008;28:382-6.

16. Munkwitz S, Elkarmouty A, Hoffmann EM, et al. Comparison of the ICare rebound tonometer and the Goldmann applanation tonometer over a wide IOP range. Graefes Arch Clin Exp Ophthalmol 2008;246:875-9.

17. Chui WS, Lam A, Chen D, et al. The influence of corneal properties on rebound tonometry. Ophthalmology 2008;115:80-4.

18. Altman DG. Method comparison studies. In: Practical statistics for medical research. Chapman \& Hall/CRC, 1991:Chap 14, 396-403.

19. Tiolat. iCare tonometer: user's and maintenance manual. Helsinki.

20. Baudouin C, Gastaud P. Influence of topical anesthesia on tonometric values of intraocular pressure. Ophthalmologica 1994;208:309-13.

21. Lundvall A, Svedberg H, Chen E. Application of the ICare rebound tonometer in healthy infants. J Glaucoma 2011;20:7-9.

22. Martinez-de-la-Casa JM, Garcia-Feijoo J, Vico E, et al. Effect of corneal thickness on dynamic contour, rebound, and goldmann tonometry. Ophthalmology 2006;113:2156-62.

23. Van der Jagt LH, Jansonius NM. Three portable tonometers, the TGDc-01, the ICARE and the Tonopen XL, compared with each other and with Goldmann applanation tonometry*. Ophthalmic Physiol Opt 2005;25:429-35.

24. Jorge J, Fernandes P, Queiros A, et al. Comparison of the IOPen and iCare rebound tonometers with the Goldmann tonometer in a normal population. Ophthalmic Physiol Opt 2010;30:108-12.

25. Poostchi A, Mitchell R, Nicholas S, et al. The ICare rebound tonometer: comparisons with Goldmann tonometry, and influence of central corneal thickness. Clin Exp Ophthalmol 2009;37:687-91.

26. Krakau CE, Wilke K. On repeated tonometry. Acta Ophthalmol (Copenh) 1971;49:611-14.

27. Hussein MA, Paysse EA, Bell NP, et al. Corneal thickness in children. Am J Ophthalmol 2004;138:744-8. 